Kieler Beiträge zur Filmmusikforschung, 1, 2008 / 26

\title{
Musik im Hindifilm zwischen Narration und Reflexion
}

\section{Claus Tieber (Wien)}

Das zentrale Kennzeichen des Hindi-Films ist die Musiknummer. Abgesehen von einigen wenigen Ausnahmen gibt es keinen Hindi-Film ohne Musiknummern. Die Bedeutung dieser Filmmusik für die indische Kultur auf dem Subkontinent und in der Diaspora ist unbestritten. Ihre narrative Funktion hingegen wird weitgehend übersehen oder geleugnet: Die Musiknummern hätten keine Verbindung zur Geschichte, die der Film erzählt, für das Verständnis der Handlung eines Hindi-Films seien die Musiknummern ohne Bedeutung, so die allgemeine Meinung, die selbst in der indischen Filmindustrie vertreten wird. So meint etwa der Drehbuchautor und Song-Texter Javed Akthar, die Musiknummern seien ,not part of the narrative“ (Kabir 1999, 130). Unbestritten ist, dass es etliche Musiknummern gibt, die tatsächlich so gut wie nichts zur Handlung beitragen. Die überwiegende Mehrheit jedoch besitzt sowohl narrative wie auch reflexive Funktionen. Das sei an einigen Beispielen genauer dargestellt.

\section{Mississippi Masala: Aber mein Herz schlägt indisch}

Uganda, 1972. Diktator Idi Amin weist alle Asiaten aus. Unter ihnen eine indische Familie, die mit dem Bus zum Flughafen fährt. Eine Polizeikontrolle hält den Bus an. Die Mutter muss aussteigen und ihren Koffer öffnen. Sie hat einen Kassettenrekorder bei sich. Die Polizisten fordern sie auf, ihn einzuschalten. Ein Lied erklingt:

Mera juta hai Japani

Ye patlun Inglistani

Sar pe lal topi Russi

Phir bhi dil hai Hindustani... 
[My shoes are Japanese,

Pantalon, English are these

On my head a red Russian hat.

But my heart, it's Indian for all that...]

Der Film, aus dem diese Szene stammt, heißt Mississippi Masala. Er wurde von Mira Nair nach einem Drehbuch von Sooni Taraporevala 1992 gedreht. Es handelt sich dabei um einen so genannten DiasporaFilm, einen Film, der in der südasiatischen Diaspora von NRIs (Non Resident Indians) spielt und das Leben in der Diaspora zum Inhalt hat. Der Umstand, dass in diesem Fall nicht Indien, sondern Uganda als „Homeland“ in Opposition zur amerikanischen Diaspora gebracht wird und sich darüber hinaus die indische Protagonistin, die Tochter jener Frau mit dem Kassettenrekorder, im Laufe des Films in einen AfroAmerikaner verliebt, ist ein Kommentar zur Konstruktion von Begriffen wie „Heimat“ und „Diaspora“. Die Verbundenheit der indischen Familie in Uganda mit Indien wird in dem relativ kurzen Auftakt des Films, der in Uganda spielt, gerade durch dieses musikalische Zitat betont. Das Lied, das hier zu hören ist, stammt aus dem Film Shree 420 (Indien 1955), in dem es der Regisseur und Hauptdarsteller Raj Kapoor „singt.“ Es gehört zu den populärsten Liedern Indiens. Es ist laut Jigna Desai „the Hindi film song quintessentially associated with diaspora“" (Desai 2004, 77).

Das Zitat dieses Liedes fungiert in Mississippr Masala als akustisches Requisit, es gibt eine zusätzliche, nichtzentrale Information über die Figur, der es zugeordnet ist. In Shree 420 ist besagtes Lied die Auftrittsnummer des Protagonisten. Raj wird damit, außerhalb einer dramatischen Szene, ohne Interaktion mit anderen Figuren ausschließlich über den Text und die Musik eingeführt. Raj Kapoor spielt in diesen Film wie in etlichen anderen einen Tramp, einen Awara, der offen an die Tramp-Figur Chaplins angelehnt ist. Die fröhliche Stimmung der Musik gibt den Charakter der Figur und die optimistische Grundstimmung der Jahre nach der Unabhängigkeit Indiens wieder. Der Text reagiert auf die im Unabhängigkeitskampf proklamierte Strategie des Swadeshi, der Ablehnung aller ausländischen, sprich: britischen Produkte. Hier behauptet jedoch eine Figur trotz der zahlreichen ausländischen Einflüsse, denen sie ausgesetzt ist, im Herzen ein Inder (resp. eine Inderin) zu sein. Dies wiederum eignet sich Jahrzehnte nach Kapoors Film perfekt als Kommentar zur Problematik in der Diaspora lebender Inderinnen und Inder, als Postulierung der Portabilität ,indischer Werte.“ Das Lied hat sich verselbständigt und seinen Platz im populären Repertoire gefunden. Die Musik, insbesondere die Filmmusik wird hier zum Ausdruck kultureller Identität bzw. den Schwierigkeiten damit. Dieser Identitätskonflikt erfüllt ursprünglich, in SHREE 420 sowohl eine expositorische als auch eine kommentative Funktion. Jahrzehnte später hat sich das Lied verselbstständigt und wird in MississiPPI Masala zum kommentierenden Element, das nur in Kenntnis des Liedes und seines Kontextes verständlich ist. 
Musiknummern in bzw. aus Hindi-Filmen können somit sowohl narrative als auch kommentative Funktionen erfüllen. Für eine praktikable Kategorisierung dieser Funktionen im Hindi-Film ist es notwendig, zunächst auf eine allgemeine Ebene $\mathrm{zu}$ wechseln. Ich möchte zwischen den konstanten Eigenschaften und Charaktermerkmalen der Figuren und deren veränderbaren Gefühlszuständen unterscheiden. Und ich möchte weiter zwischen den Handlungsfluss unterbrechenden und beschleunigenden Funktionen von Musiknummern differenzieren. Die vier Funktionsbereiche, die so entstehen, sind keineswegs strikt voneinander getrennt, sondern überlagern einander.

\section{Die Musiknummer als Exposition einer Figur}

Die Einführung einer Figur mittels Auftrittslied ist ein im Hindi-Film durchaus gebräuchliches Mittel. Das Star-Image von Bollywood-Schauspielern ist mit derartigen Liedern auf das Engste verbunden, auch wenn diese ihre Lieder nicht selbst singen. Einzig Amitabh Bachchans Star-Image lässt sich nicht mit Musik, sondern besser mit Dialog auf den Punkt bringen. Raj Kapoor jedoch wird immer mit besagter Nummer verbunden sein.

\section{Produktionsweise}

Die Musik (einschließlich des Liedtextes) erfüllt hier die Funktion der Einführung einer Figur, die in westlichen Filmen fast immer dem Drehbuch (resp. der Handlung) zukommt. Darum ist es an dieser Stelle nötig, auf die spezifische Produktionsweise des Hindi-Films zu verweisen. Nicht nur singen die Schauspieler nicht selbst - Hindi-Filme werden sogar zur Gänze synchronisiert. So etwas wie einen authentischen Soundtrack im Unterschied zum musikalischen gibt es somit nicht. Das Drehbuch eines Hindi-Films existiert zu dem Zeitpunkt, zu dem der Music Director für den Film engagiert wird, nur in rudimentären Zügen. Der Music Director, der Komponist des Films, wird die Geschichte des Films in der Regel nicht lesen, sondern erzählt bekommen. Die Musiknummern werden geschrieben, bevor mit den Dreharbeiten begonnen wird, sie stehen im Drehplan am Beginn des Films.

Der zweite wichtige Punkt ist die weitgehend fehlende Integration der Musiknummern in die Narration des Films. D.h. ein Hindi-Film macht sich in der Regel keine Mühe, eine Musiknummer diegetisch zu begründen. Singen und Tanzen sind Konventionen des Hindi-Films und müssen nicht realistisch motiviert werden. Ein Umstand, der in den letzten Jahren eine deutliche Änderung zugunsten stärker integrierter Musiknummern erfahren hat. 


\section{Diegetische Musiknummern}

Neben jenen Nummern, die wie etwa Traumszenen nur lose in die Diegese des Films integriert sind, gab es immer schon solche, die durch die Diegese des Films begründet werden. So eignet sich der Umstand, dass in Indien sowohl Gebete wie auch Gedichte zumeist gesungen werden, für ihre Sonderstellung in einer filmischen Form, in der Musiknummern zur Konvention gehören. Schriftsteller finden sich in Filmen wie Pyaasa (1957) oder Kнавнie Khabhie (1976), singende Heilige stehen im Mittelpunkt der religiösen Filme (devotionals) der 1920er und 1930er Jahre. Religiöse Feste, die eine weitere Möglichkeit eröffnen, Musiknummern mit diegetischen Funktionen in die Erzählung zu integrieren, sind seit den 1990er Jahren verstärkt zu sehen. Häufig treten professionelle Sänger als Figuren von Filmen auf. Dabei handelt es sich zumeist um Ghazalsänger in einem muslimischen Umfeld, die je nach Zeit und politischer Intention der Filmemacher als integraler Bestandteil der indischen Familie wie etwa in Amar, Aквнar, Anthony (1977) oder als mit Pakistan gegen die Interessen Indiens arbeitende Verschwörer wie in SARFArosh (1999) dargestellt werden. In jedem Fall sind die Lieder dieser Figuren diegetisch motiviert. Auch Sängerinnen stehen im Hindi-Film zumeist in einem muslimischen Umfeld. Die in der Öffentlichkeit der erzählten Welt auftretende, singende und tanzende Frau ist in der Regel eine Kurtisane oder Prostituierte.

\section{Musikalische Bezüge zu den Mahabharata und Ramayana}

Die Musik im Hindi-Film vermittelt Basisinformationen über die Figuren. Die beiden zentralen Dichotomien des Hindi-Films werden recht eindeutig auch musikalisch gekennzeichnet:

- Indisch vs. Westlich und

- Hindu vs. Moslem.

In der Instrumentation und im Rhythmus werden die Unterschiede manifest gemacht, wobei die islamische musikalische Tradition nie negativ dargestellt wird; darin unterscheiden sich mitunter Musik und Figuren letztere können durchaus als antagonale Figuren, als Bösewichte und Schurken auftreten, ohne dass daraus ein Urteil über die ihnen assoziierte Musik ableitbar wäre.

Figuren sind im Hindi-Film weit weniger psychologisch motivierte Charaktere als man sie aus westlichen Filmen kennt. Sie stehen zumeist für größere Ideen und Ideale, sie stehen in einer Verbindung bzw. werden in Verbindung gebraucht mit den beiden großen Epen Indiens, mit dem Mahabharata und dem Ramayana. Auch hier spielt wieder die Musik eine manchmal zentrale Rolle - sie verweist als spezifisch indische Form der Intertextualität auf eine Erzählung außerhalb der filmischen Erzählung. So werden immer wieder bewusste Verweise in die kulturelle Tradition gemacht, durch die Musiknummern, aber auch durch 
entsprechende Anspielungen in der Namensgebung der Figuren sowie in expliziten Dialogpassagen.

Als Beispiel für eine Musiknummer, die sowohl auf einen mythologischen Text wie auch auf Allgemeinwissen in Form von Klatsch und Tratsch, das zur Produktionszeit des Films bekannt war, verweist, dient mir Jo tum tora piya aus Silsila (1981). Der Film behandelt das für den Hindi-Film seltene Thema der ehelichen Untreue. In einer Dreierkonstellation, die wiederum recht typisch für den Hindi-Film ist, steht der von Bollywood-Star Amitabh Bachchan gespielte Amit zwischen zwei Frauen. Er heiratet Shoba (Jaya Baduri), ist aber nach wie vor in Chandni (Rekha) verliebt. Besagte Musiknummern findet sich im Film unmittelbar nachdem Amit Shoba verlassen hat.

Jo tum toro piya $[\ldots]$

[...Meera thus says to Krishna the Lord

I am your servant. You are my God.

O precious, even if you leave me, I can't leave you.]

Während des Liedes entfernen sich die Eheleute buchstäblich voneinander: Während sie an ihrem Platz bleibt, fährt der Mann mit seiner Geliebten fort, recht weit, wie der Schnee, der das Szenario bedeckt, impliziert. Die Landschaft ist hier, im Unterschied zu anderen Musiknummern im Hindi-Film, diegetischreal und keine Phantasie-Landschaft. Das Lied bringt die Figur der Shoba explizit in Verbindung mit Meera, mit der in Indien bekannten Geschichte des untreuen Krishna, gibt so eine Interpretationshilfe, die sich aus der Geschichte des Films allein nicht zwingend ergibt. Eingeleitet wird das Lied mit dem Solo einer Bambusflöte. Schon die Wahl des Instruments, die Bambusflöte oder Bansuri, verweist auf den folgenden Inhalt des Liedes - die Bansuri gilt als Instrument Krishnas.

Neben dieser religiösen Ebene spielt in dieser Musiknummer wie im ganzen Film auch der Umstand mit, dass die Hauptdarsteller, Amitabh Bachchan und Jaya Baduri, zum Zeitpunkt der Dreharbeiten bereits einige Zeit miteinander verheiratet waren, und in der Öffentlichkeit über eine außereheliche Affäre Bachchans mit eben jener Rekha gemunkelt wurde, die im Film die Geliebte Bachchans spielt. Dieser weit verbreitete Klatsch und Tratsch gab dem Film und damit auch dieser Szene eine zusätzliche Bedeutungsebene. Gregory Booth fasst dies so zusammen: „The musical scene in which ,Jo tum toro' appears expresses the emotional core of both the real and the cinematic stories in a language that combines religion, gossip, narrative and musical conventions“ (Booth 2000, 139). 


\section{Musik in der Zeit}

Musiknummern im Hindi-Film können nach ihren Funktionen für den Handlungsfluss unterschieden werden. Sie können ihn stoppen, die im Film vergehende Zeit anhalten. Eine derartige Retardation wird den Musiknummern im Hindi-Film gemeinhin als Grundfunktion zugeschrieben. Musikalische Traumsequenzen etwa unterbrechen den Handlungsfluss, die Handlung setzt danach an dem Punkt wieder ein, an dem die Musiknummer begonnen hat. Es ist, als wäre nichts geschehen. Und dennoch ist das Publikum über die Wünsche und Träume, über den Gefühlshaushalt der Figur danach besser informiert als zuvor.

Musiknummern können aber auch im Hindi-Film größere Ellipsen überbrücken, längere Zeitspannen bebildern und akustisch gestalten. Sie können somit die diegetische Zeit beschleunigen. Das Vergehen mehrerer Jahre, das Erwachsenwerden von Kindern, ein räumlicher Wechsel der Handlung, der mit dem Vergehen von Zeit verbunden ist, all das ist mit Musiknummern darstellbar. Auch im westlichen Film sind vergleichbare Montagesequenzen, in denen vor allem die Musik für die Kontinuität sorgt, gebräuchlich. Eine derartige Überbrückung einer längeren Zeitspanne kann und wird auch zur Vertiefung der Thematik des Films dienen.

Bомвау (1995) ist die erste Liebesgeschichte des indischen Films zwischen einem Hindu und einer Muslima. Diese Liebesgeschichte spielt vor dem Hintergrund der Ausschreitungen in Bombay, die der Zerstörung der Babri Moschee in Ayodhya folgten. In der Musiknummer Dil Hua Hai Deewana wird gleich zu Beginn die Information gegeben, das Shaila schwanger ist. In der an die Musiknummer anschließenden Szene ist in einem Dialog der beiden Väter des Liebespaares zu erfahren, dass neun Monate vergangen sind. Die Szene vermengt Bilder des alltäglichen Lebens mit visuellen Zeichen der beiden Religionen. Wobei diese Zeichen, und das ist das Besondere an dieser Szene, nicht eindeutig den Figuren zugeschrieben werden, sondern variabel, austauschbar sind. So trägt der Hindu Shekar ein Skull Cap, eine weiße Kopfbedeckung, wie sie Moslems tragen. Shaila, die Muslima, trägt in genau dieser Einstellung einen orangenen Sari, der Farbe des Hinduismus. Shaila betet (wie eine Muslima) und trägt sich den roten Punkt als Zeichen der Ehe mit Shekar auf (wie eine Hindu-Frau). Den Schleier, der ihr Gesicht noch am Beginn des Films verdeckte, hat sie abgelegt. Wir sehen ein glückliches inter-religiöses Paar, das erste in der Geschichte des indischen Films. Die Lyrik des Liedes betont noch einmal die zentrale Aussage des Films: „We have but one god, so let's be one too. [...] Live your live and let other live.“ Was sich so banal anhört, ist angesichts des realen zeitgenössischen Hintergrunds der Geschichte, des gegenseitigen Mordens von Hindus und Moslems 1992/93, keineswegs selbstverständlich. Man hört eine weibliche Stimme, die wohl eindeutig Shaila zugeordnet werden kann, sowie einen Kinderchor, der die zu erwartenden Zwillinge vorwegnimmt. Frauen und Kinder als sozusagen ,unschuldige“ Stimmen verstärken die pazifistische Botschaft der Musik-Nummer und des Films. 
Wie das Bild religiöse Zeichen mixt, so sind auch in der Musik verschiedene Einflüsse zu hören. Der Song beginnt mit einer Bassline auf einem geslappten E-Bass, klassische indische Instrumente wie die Sitar kommen hinzu. Diese Musiknummer verbindet in recht kurzer Zeit unterschiedliche musikalische und visuelle Zeichen, sie macht damit gerade die Hybridität zu einem der charakteristischen Merkmale Indiens und des populären indischen Films, wobei sie ausdrücklich als positives Element, als Ideal, als Traum gekennzeichnet ist und somit in der Tradition derartiger Musiknummern steht. Gleichzeitig ist dieser Idealzustand der Harmonie der Religionen deutlich gegen den Rest des Films, in dem es um die blutigen Auseinandersetzungen zwischen Hindus und Moslems geht, gesetzt. Der Film zeigt die Realität der Gewalt und des Hasses. Doch für wenige Minuten ist es eine Musiknummer, die eine positive Utopie zu zeigt.

Die Musiknummer, die hier zunächst als Überbrückung eines Zeitsprungs motiviert ist, wird so zur Essenz des Films, the whole message in a nutshell. Solcherart können Konventionen des Hindi-Films unter Beibehaltung ihrer primären narrativen Funktionen, in dem Fall der Zeitraffung, zusätzliche kommentierende und vertiefende Bedeutung erhalten.

\section{Recap, remixed}

Eine Besonderheit des indischen Film stellen visuelle Wiederholungen dar, die mit Musik unterlegt sind. Auch der westliche Film kennt derartige Recaps, wenngleich sie nicht so häufig zu finden sind wie im HindiFilm. Eine Besonderheit stellt jedoch eine Variante dieser Zusammenfassung dar, in der nicht die Handlung, wie sie war, gezeigt wird, sondern wie sie sein hätte können bzw. wie eine mögliche Zukunft aussehen könnte. In Dilwale Dulhania Le Jayenge (1995) etwa wird im zweiten Teil des Films jene Europareise, welche die Protagonisten im ersten Teil des Films machen, noch einmal zusammengefasst gezeigt, mit dem kleinen aber markanten Unterschied, dass das Liebespaar des ersten Teils in der Zusammenfassung verheiratet ist, was am roten Sari der Protagonistin ablesbar ist. Die rückblickende Zusammenfassung wird so zur traumhaften Vorblende. Musikalisch bedient sich die Sequenz eines bereits aus dem ersten Teil bekannten Motivs.

Eine Musiknummer kann sich aber auch in der diegetischen Zeit des Films bewegen. Mitunter finden wichtige Ereignisse mitten in Musiknummern statt. So wird etwa in Amar, Akbar, Anthony die blinde Mutter der drei im Titel genannten Brüder mitten in einer Musiknummer wieder sehend. 
Musiknummern im Hindifilm können sowohl kommentative als auch narrative Funktionen erfüllen. Sie holen über Verweise und Ankoppelungen an extradiegetische Texte vorzugsweise aus einem der beiden großen indischen Epen, dem Mahabharata und dem Ramayana, eine zusätzliche Ebene in den Film ein, was mitunter schon über musikalische oder liedtextliche Zitate geleistet werden kann. Auf der narrativen Ebene werden über derartige Verweise auch narrative Informationen gegeben, die sowohl die Charakterisierung einer Figur als auch den Handlungsverlauf und dessen Interpretation betreffen kann. Beide Funktionen, kommentative und narrative, sind keineswegs streng säuberlich voneinander getrennt, sondern können sich in ein und derselben Musiknummer finden. Auch was den Fortgang der Handlung betrifft, kann eine Musiknummer diesen sowohl zunächst stoppen, um ihn sodann sogar zu beschleunigen.

\section{Die Qawwali Party}

Eine Besonderheit des Hindi-Films ist seine Trennung von Bild und Ton. Der gesamte Film wird (oder wurde bis vor kurzem) nachsynchronisiert. Playback bei Musiknummern gibt es seit 1935, die Sängerinnen und Sänger des Hindi-Films sind mitunter bekannter als so mancher Star. Auf der Ebene der Diegese des Films stellt sich schnell die Frage: Wer singt hier eigentlich? Ist in der westlichen Tradition des Musikfilms die Solidarität von Stimme und Figur in der Regel eingehalten, so ist diese Bindung im Hindi-Film brüchiger und wird von dramaturgischen Konventionen überlagert, welche die Stimme verschiedenen dramatischen Instanzen zuordnet. Es ist also keineswegs so, das die singende Stimme automatisch und immer als zu der Figur gehörend angesehen werden kann, die gerade zu sehen ist.

Eine besondere Rolle kommt dabei in etlichen Hindi-Filmen einem Chor zu, der als solcher auch zu sehen ist. Die Darsteller des Chors sind keine Figuren des Films, sie sind weder zuvor noch danach zu sehen. Es handelt sich dabei um keinen Chor in der buchstäblichen Bedeutung des Wortes, sondern um eine Qawwali Party, eine Gruppe von Musikern und Sängern, die ein Qawwali, eine spezifische musikalische und literarische Form, singen. Als Chor bezeichne ich die Party nicht nur in einem musikalischen Sinn, sondern vor allem in Hinsicht auf ihre dramatische und narrative Funktion, die dem eines Chores in der griechischen Tragödie gleicht. Das individuelle Geschehen wird auf eine Ebene allgemeiner Bedeutung gehoben. Dazu später mehr.

Besonders deutlich wird die Sonderstellung des Chors in der Musiknummer Nahi Hina Tha aus PARDES (Indien 1997). Der Film schildert eine typische Dreiecksgeschichte: Ein in den USA reich gewordener Patriarch will seinen Sohn mit der in Indien lebenden Ganga verheiraten. Sein Ziehsohn Arjun soll alles vorbereiten. Im Laufe des Films verlieben sich Arjun und Ganga ineinander, der vorgesehene Bräutigam 
entpuppt sich als verwestlicht und unmoralisch. Die Liebe zwischen Arjun und Ganga ist aber eine verbotene, die gegen die Autorität ihrer Väter bzw. Stiefväter verstößt. In besagter Musiknummer ist eine Qawaali Party zu sehen. Eine männliche Stimme eröffnet den Gesang. Dann setzt eine weibliche Stimme ein. Die Sängerin ist inmitten weiterer Frauen zu sehen. Eine Qawaali Party besteht traditionsgemäß ausschließlich aus Männern. Ihr wird hier, wie in vielen Hindi-Filmen, eine weibliche Gruppe gegenübergestellt, die räumlich von der männlichen getrennt ist. Es gibt keine einzige Einstellung, in der sie gemeinsam zu sehen sind. Während sie visuell getrennt sind, vereint sie die Musik, sie singen beide dasselbe Lied, folgen derselben Melodie. Der Sänger setzt fort. Nun übernimmt die weibliche Stimme die Melodie. Während die männliche Stimme in der dritten Person gesungen hat, beginnt die weibliche nun, den Text in der ersten Person vorzutragen. Dieser Wechsel von dritter zu erster Person innerhalb eines Liedes, vom allgemeinen Kommentar zum individuellen Statement, ist für den Hindi-Film typisch und hat eine lange Tradition. Das Liebespaar des Films ist erst jetzt zu sehen, die beiden kommen sich näher, wenngleich sie im Vorangehen der Handlung noch nicht zusammenkommen dürfen. Ganga erinnert sich an vorangegangene Szenen. Da ertönt eine andere männliche Stimme, die das Publikum nur als die von Arjun interpretieren kann. Eine Qawaali hat traditionellerweise zwei individuell vom Chor abgesetzte Sänger. Nach einer kurzen Dialogszene in der die Musik, der Gesang bleibt im Hintergrund, folgt wieder die erste männliche Stimme der Party. Die Musik widerspricht dem Dialog, in dem Arjun seine Gefühle für Ganga bestreitet. Die Musik macht die wahren Gefühle der Figur hörbar. So vage und allgemein die Musik und die Texte auf Grund der Produktionsweise sind, so unmittelbar nehmen sie doch Bezug auf den Punkt der Handlung, der an dieser Stelle erreicht ist: jetzt ist es Zeit für eine Entscheidung. Die Handlung des Films, die spätestens mit der Begegnung der beiden Liebenden wieder fortgeschritten ist, nimmt während des Liedes ihren weiteren Verlauf. Die finale Konfrontation steht kurz bevor, die beiden Seiten beziehen Stellung.

Figuren und Singstimmen sind in dieser Nummer getrennt. Wer also singt hier? Der Verweis auf den antiken griechischen Chor scheint angebracht. Er ist nicht einfach die Stimme des Dichters, wie Siegfried Melchinger schreibt, genauso wenig wie die Stimme des Publikums: „Einer seiner wichtigsten Funktionen besteht [...] darin, die Gestimmtheit, die sich aus einer dramatischen Situation ergibt, durch das Medium der emotionalen Musik mit Gesang und Tanz gleichsam in die Stimmung des Zuschauers zu ,transportieren', oft sind in die Gesänge tiefe Gedanken eingesenkt, die auf dem gleichen Wege dem Nachdenken des Zuschauers übermittelt werden“ (Melchinger 1990, 65). Eine ähnliche Sonderstellung hat der Chor in zahlreichen Musiknummern des Hindi-Films. Eine Qawwali Party fungiert in besagter Szene als Chor, die Texte heben die „Gestimmtheit“ der Szene auf eine höhere Ebene, die oft religiös eingefärbt ist. In PARDES ist die Musik traditionell religiös konnotiert, was zu besagten ,tiefen Gedanken“ zusätzlich animieren mag. Der Chor ist sowohl innere Stimme als auch objektive kommentierende Stimme. Kommentierend in genau dem Sinn, den Melchinger angesprochen hat: Die dramatische Situation wird verallgemeinert. In der Tradition der UrduLyrik ist gerade in Qawwalis ist der Gebrauch erotischer Metaphern für religiöse Themen gebräuchlich, was 
sich wiederum der Hindi-Film in den Formen, in denen er diese ursprünglich nicht-filmische Ausdrucksform gebraucht, zu Nutze macht. Und dabei den religiösen Gehalt zunehmend verliert, wie manche Kritiker anmerken.

Diese Qawaali-Nummer ist nicht nur kommentierend und damit handlungsunterbrechend, sondern auch dialogisch und die Handlung vorantreibend. Während der ca. sechsminütigen Szene kommen alle Figuren auf den Schauplatz der Szene, ob von nah oder von fern (USA), die beiden Konfliktparteien bringen sich in Aufstellung und das Publikum wird über Gangas und Arjuns wahre Gefühle informiert. Schon der griechische Chor konnte in Dialog mit den Figuren des Stücks treten, und auch hier scheint es zwischen den jeweils ersten und zweiten Stimmen so etwas wie einen Dialog zu geben, ein Einsehen, ein Bewusstwerden der Figuren um die Bedeutung ihrer Situation.

\section{Summa}

Musiknummern im Hindi-Film haben nicht nur eine seit Jahrzehnten geläufige Konvention, sie erfüllen auch bestimmte narrative und reflexive Funktionen. Einige dieser Funktionen haben sie mit Filmmusik in westlichen Filmen gemeinsam, einige mit Song-and-Dance-Szenen im Hollywood-Musical. Damit eröffnen sich zwei dramaturgisch essentielle Funktionen:

- die Möglichkeit des Verweises auf die beiden bekanntesten indischen Erzählungen, auf das Mahabharata und das Ramayana,

- sowie die Verwendung eines Chors bzw. von Stimmen mit chorischer Funktion in einem den Funktionen des Chors in der attischen Tragödie verwandten Form.

Gerade die Qawwali-Nummern sind ein eigenes Formstück des Hindi-Films, das sich so in keiner anderen nationalen Kinematographie finden lässt. Sie machen noch heute das Besondere des Hindi-Films aus.

Ob und inwieweit sich dies in den nächsten Jahren auf Grund ökonomischer und technischer Veränderungen (neue Finanzierungsquellen, Digitalisierung) verändern wird, bleibt abzuwarten.

\section{Literatur}

Booth, Gregory (2000) Religion, gossip, narrative conventions and the construction of meaning in Hindi film songs. In: Popular Music 19,2, 2000, S. 125-145.

Desai, Jigna (2004) Beyond Bollywood. The Cultural Politics of South Asian Diasporic Film. New 
York/London: Routledge.

Kabhir, Nasreen Munni (1999) Talking films. Conversations on Hindi Cinema with Javed Akhtar. New Delhi: Oxford University Press.

Melchinger, Siegfried (1990[1974]) Das Theater der Tragödie. Aischylos, Sophokles, Euripides auf der Bühne ihrer Zeit. München: Deutscher Taschenbuch Verlag.

Tyrell, Heather / Dudrah, Rajindeer (2006) Music in the Bollywood Film. In: Conrich, Ian und Tincknell, Estella (eds.): Film's Musical Moments. Edinburgh: Edinburgh University Press, S. 195-208.

\section{Filmographie}

Amar, Aкbhar, Anthony (Indien 1977, Manmohan Desai).

Awara (Indien 1951, Raj Kapoor).

Bombay (Indien 1995, Mani Rantnam).

Dilwale Dulhania Le Jayenge (Indien 1995, Aditya Chopra).

Jis Desh Mein Ganga Behti Hai (Indien 1961, Raj Kapoor).

Khabhie Khabie (Indien 1976, Yash Chopra).

Kuch Kuch Нота Hai (Indien 1998, Karan Johar).

Mississippi Masala (USA 1991, Mira Nair).

Pardes (Indien 1997 Subhash Ghai).

PyaAsa (Indien 1957, Guru Dutt).

Sarfarosh (Indien 1999, John Matthew Matthan).

SHREe 420 (Indien 1955, Raj Kapoor).

SILSILA (Indien 1981, Yash Chopra).

\section{Empfohlene Zitierweise:}

Claus Tieber: Musik im Hindifilm zwischen Narration und Reflexion.

In: Kieler Beiträge zur Filmmusikforschung 1, 2008.

URL: http://www.filmmusik.uni-kiel.de/beitraege.htm

Datum des Zugriffs: 1.2.2008.

Kieler Beiträge zur Filmmusikforschung (ISSN 1866-4768)

Copyright $(\mathrm{C}$ by Claus Tieber. All rights reserved.

Copyright (C) für diese Ausgabe by Kieler Gesellschaft für Filmmusikforschung. All rights reserved.

This work may be copied for non-profit educational use if proper credit is given to the author and „Kieler Beiträge für Filmmusikforschung“". 